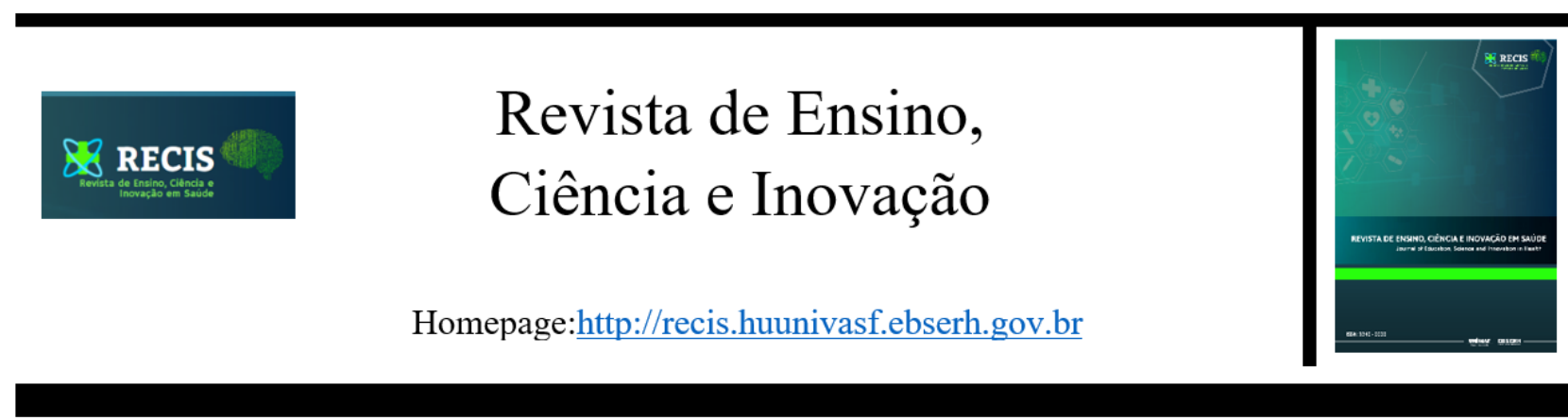

\title{
Análise da qualidade de sono e da preferência diurna dos trabalhadores em turnos da área da saúde
}

\section{Analysis of sleep quality and daytime preference of health shift workers}

\author{
Pedro Victor Freitas Medrado ${ }^{1}$, Alane Mota dos Santos ${ }^{1}$, Bruna Del Vechio Koike ${ }^{1}$ \\ ${ }^{1}$ Colegiado de Medicina, Universidade Federal do Vale do São Francisco, Petrolina/PE
}

Autor correspondente: pedro.medrado@ discente.univasf.edu.br

Artigo recebido em 23/10/2020 e aceito em 08/12/2020

\begin{abstract}
RESUMO
Os profissionais da saúde estão sujeitos a trabalhos em turnos em horários não convencionais, o que muitas vezes, pode não coincidir com seus ritmos circadianos endógenos. Neste estudo, o objetivo foi investigar de qual modo a preferência diurna associa-se com os padrões do ciclo sono e vigília, em especial a qualidade do sono e da sonolência diurna excessiva. Para isso, foram aplicados questionários específicos para descrever os turnos de trabalho e para avaliar os parâmetros de sono. Utilizamos o questionário de Pittsburgh para avaliar a qualidade de sono, o de Epworth para avaliar a sonolência diurna e o HO para a preferência diurna. Os testes t de Student, de correlação de Pearson e de Análise de variância (ANOVA) foram utilizados a fim de analisar os parâmetros de sono dos trabalhadores em turnos de hospitais. A amostra constituiu-se de 59 indivíduos, de ambos os sexos, que trabalhavam no regime $12 \mathrm{~h}$ de trabalho intercalados com $36 \mathrm{~h}$ de folga em turnos diurnos ou noturnos. Verificamos que (i) a preferência diurna individual não é levada em conta na atribuição do turno; (ii) trabalhadores de ambos os turnos apresentam má qualidade de sono e (iii) queixas de sonolência diurna semelhantes. Entretanto, encontramos correlação entre a má qualidade de sono e a sonolência diurna excessiva somente entre os trabalhadores noturnos.

Palavras-chave: Sono; Cronobiologia; Medicina do trabalho; Pessoal de saúde; Trabalho em turnos.
\end{abstract}

\begin{abstract}
Health professionals work at unconventional hours, which may not coincide with their endogenous circadian rhythms. In this study, the objective was to investigate if daytime preference is associated with patterns of sleep and wake cycle, sleep quality and excessive diurnal sleepiness. For this, specific questionnaires were used to evaluate work shifts and to assess sleep parameters. We used the Pittsburgh questionnaire to assess sleep quality and Epworth's to assess diurnal sleepiness. The T-Student, Pearson correlation tests and Analysis of variance (ANOVA) were used to analyze the sleep parameters of hospitals workers. The sample was composed by 59 individuals, both sexes, with $12 \mathrm{~h}$ of work interspersed with 36 hours off in a diurnal or nocturnal shift. We found that (i) individual daytime preference is not taken into account when assigning the nocturnal or diurnal shift; (ii) workers on both shifts have similar quality of sleep and (iii) complaints of daytime sleepiness were similar. However, we found a correlation between poor sleep quality and excessive diurnal sleepiness only among night workers.
\end{abstract}

Keywords: Sleep; Chronobiology; Occupational Medicine; Health Personnel; Shift Work Schedule. 


\section{INTRODUÇÃO}

A sociedade vem se desenvolvendo há milênios, de forma que grande parte das atividades são realizadas na presença da luz solar, como a pesca, o cultivo e a caça. Essa sincronização aos ciclos ambientais provoca o alinhamento dos eventos fisiológicos circadianos e nos leva a manter a ordem temporal interna, com as relações de fase bem estabelecidas entre as variáveis fisiológicas ${ }^{1-4}$.

Atualmente, os serviços que são considerados essenciais, como os trabalhos relativos à saúde, necessitam de uma continuidade das atividades pelas $24 \mathrm{~h}$ do dia. Com isso, surge a demanda de profissionais que cumpram as jornadas de trabalho em todos os horários, diurnos e noturnos. Quando a atividade de trabalho é noturna, implica ficarem acordados e alertas, em um horário no qual grande parte da humanidade está dormindo ${ }^{5,6}$. Por outro lado, repousam durante o dia, horário no qual a grande maioria está em atividade $^{7}$.O trabalho noturno exige que o indivíduo inverta o horário da fase de sono e o aloque em um horário não convencional. Isto requer uma adaptação do trabalhador, pois necessita, além de inverter a sua fase ativa, também ressincronizar as outras variáveis fisiológicas endogenamente ${ }^{6,8,9}$. Ou seja, deve realinhar todo o seu sistema de temporização circadiano.

O termo "ritmo circadiano" foi criado por Halberg para descrever oscilações endógenas em organismos, que foram assim classificadas por apresentar um período aproximado ao da rotação diária do planeta Terra em torno de $\mathrm{si}^{10}$. A relação entre a saúde humana e os ritmos circadianos é bidirecional: tanto o sistema de temporização circadiano coordena o ritmo das nossas variáveis fisiológicas, quanto o nosso comportamento - de trabalho, de alimentação - em horários irregulares causa ruptura nos ritmos, levando a um conflito temporal e provocando um desalinhamento crônico $^{11}$.

O sistema de temporização endógeno sincroniza-se com os ciclos ambientais através de pistas temporais. A pista temporal que exerce maior efeito no nosso oscilador central é o ciclo claro e escuro ambiental ${ }^{12}$. As projeções diretas da retina para os núcleos supraquiasmáticos (NSQ) do hipotálamo, faz com que esse oscilador central mantenha-se sincronizado aos ritmos ambientais ${ }^{13}$. Os NSQ apresentam projeções diretas e indiretas para os principais alvos de controle fisiológico e do comportamento no sistema nervoso central ${ }^{14}$. Além disso, por via humoral, este controle é mediado pela secreção de melatonina pela glândula pineal, cujo ritmo é também controlado pelo NSQ polissinapticamente ${ }^{15}$.

Nos mamíferos, um pulso de luz no início da noite, provoca atrasos no ritmo circadiano, enquanto no final da noite, o mesmo pulso de luz pode provocar avanços. Estes avanços e atrasos são os mecanismos que permitem a sincronização dos organismos ao dia e a noite ${ }^{16}$. Contudo, a capacidade de sincronização depende de fatores individuais e ambientais, como o período endógeno circadiano e a capacidade de perceber os estímulos ambientais. A principal influência externa consiste na duração e na intensidade do estímulo luminoso, além das pistas sociais ${ }^{17}$. Para os trabalhadores do turno noturno, a duração da exposição à luz é aumentada, visto que, mesmo na fase escura do dia (à noite), eles estão expostos à iluminação intensa, principalmente nas unidades de terapia intensiva (dados ainda não publicados).

As queixas de distúrbios de sono são frequentes entre eles, e isto tem uma grande implicação na ocorrência de acidentes e no afastamento do trabalho ${ }^{18}$. O controle do ciclo sono e vigília ocorre por dois mecanismos, o mecanismo homeostático e o mecanismo circadiano ${ }^{19}$. Os mecanismos homeostáticos determinam que temos uma necessidade de sono que é guiada por fatores genéticos, metabólicos e comportamentais e que vão estabelecer a duração do sono.

Por outro lado, o componente circadiano determina a fase de alocação temporal do sono. Em conjunto, esses mecanismos determinam que cada indivíduo possui um padrão de sono e uma preferência particular pelos horários de dormir e $\operatorname{acordar}^{20}$. Essa possibilidade de preferência pela alocação temporal da fase de comportamentos é denominada cronotipo ou preferência diurna, no contínuo matutinidade-vespertinidade, usualmente mensurada por uma versão traduzida do Morningness-Eveningness Questionnaire (MEQ), de Horne e Östberg $(\mathrm{HO})^{21}$. Esse questionário distingue basicamente cinco grupos: extremamente matutinos, moderadamente matutinos, extremamente vespertinos, moderadamente vespertinos e intermediários ${ }^{21}$. Em nosso estudo houve a separação em três quartis, obtidos pela 
curva normal de distribuição: matutinos, intermediários e vespertinos. A preferência por dormir cedo e acordar cedo é comum em matutinos, enquanto dormir tarde e acordar mais tarde, é comum em vespertinos ${ }^{22}$.

Conforme o descrito anteriormente, a condição de trabalho em turno pode promover alterações nos episódios de sono, podendo refletir na duração do sono, bem como na sua qualidade e, consequentemente, gerar sonolência diurna. Por outro lado, sabemos que alguns trabalhadores apresentam uma certa tolerância ao trabalho noturno, e isto nos leva a procurar quais são os fatores que fazem com que alguns se adaptem ao trabalho em turno. Neste trabalho, avaliamos as variáveis do ciclo sono e vigília nos trabalhadores de ambos os turnos da área de saúde, incluindo a preferência diurna, a sonolência excessiva e a qualidade do sono, e buscamos comparar as características apresentadas entre os indivíduos dos diferentes turnos de trabalho.

\section{MATERIAL E MÉTODOS}

O estudo, do tipo transversal, foi realizado com indivíduos que trabalham no regime $12 \mathrm{~h}$ de trabalho intercalados com $36 \mathrm{~h}$ de folga no Hospital Universitário Dr. Washington Antônio de Barros (Hospital da Universidade Federal do Vale do São Francisco) em Petrolina-PE. Segundo o setor de gestão de pessoas, cerca de 600 funcionários compõe o quadro do hospital, do qual dois terços são mulheres. Todos os trabalhadores do hospital foram convidados a responder os questionários, de modo a termos uma amostra fidedigna. Cerca de $10 \%$ dos funcionários responderam os questionários online espontaneamente e compuseram a nossa amostra. Os critérios de inclusão utilizados foram: ter histórico de trabalho em turno regular há pelo menos um ano e não fazer uso de substâncias psicoativas e fumo. Os indivíduos que possuíam diagnóstico de doenças crônicas foram excluídos do estudo.

Foi aplicada uma série de questionários para a triagem e avaliação destes indivíduos. O primeiro deles foi um questionário com perguntas sobre a situação pessoal, os hábitos sociais e de trabalho. Para a determinação do cronotipo, que reflete a preferência diurna do indivíduo, foi aplicado a versão em português do questionário Morning-Eveningness Questionnaire ${ }^{23}$, o qual chamamos de questionário HO. Este questionário atribui pontuação para 19 questões e gera um valor numérico referente aos horários de preferência diurna, que varia entre 16 e 86 pontos. Com o resultado desse questionário, classificamos em quartis conforme as respostas obtidas, o que dividiu os indivíduos nos cronotipos: matutinos (pontuação entre 75 - 59), intermediários $(58-42)$ e vespertinos (41 e 27).

A sonolência diurna foi mensurada pelo questionário de Epworth Sleepiness Scale ${ }^{24}$. Este questionário avalia a possibilidade de cochilar em 8 situações cotidianas, numa escala de zero a três, na qual 0 corresponde a nenhuma e 3 , a grande probabilidade de cochilar. Utilizando uma pontuação >10 como ponto de corte, é possível identificar indivíduos com sonolência diurna excessiva. Pontuações maiores de 16 é indicativo de sonolência grave, enquanto as baixas pontuações em pacientes com insônia são consistentes com as evidências de que esses pacientes possuem baixa propensão para adormecer, mesmo quando estão relaxados.

Utilizamos também o questionário Pittsburgh Sleep Quality Index (PSQI) ${ }^{25}$, que avalia a qualidade do sono em relação ao último mês, combinando informações quantitativas e qualitativas. O questionário consiste em 19 questões, que são agrupadas em sete componentes, com pesos distribuídos numa escala de 0 a 3 . Os componentes são: a qualidade subjetiva do sono, a latência para o sono, a duração do sono, a eficiência habitual do sono, os transtornos do sono, o uso de medicamentos para dormir e a disfunção diurna. As pontuações destes componentes variam de 0 a 21 , inversamente proporcionais: quanto maior a pontuação, pior a qualidade de sono.

Para a análise dos dados, utilizamos o SOFTWARE IBM SPSS STATISTICS BASE 22 - DATA MINING AND STATISTICAL SOLUTIONS para efetuar as análises descritivas, estatísticas e de correlação dos dados. O teste $t$ de Student foi utilizado na comparação dos escores médios dos construtos considerados, no estudo, em função das categorias de participantes. O teste de ANOVA foi utilizado para avaliar a variância dos dados nos grupos amostrais e os testes de correlação na avaliação da correspondência de uma variável sobre a outra.

O estudo foi aprovado pelo comitê de Ética em Pesquisa com Seres Humanos da Universidade Federal do Vale do São Francisco (Certificado de Apresentação para Apreciação Ética: 08369018.2.1001.5196 - UNIVASF) e os sujeitos assinaram o Termo de Consentimento Livre e Esclarecido para responderem aos questionários.

\section{RESULTADOS}

Um total de 59 questionários foram analisados. Houve a presença majoritária de 
mulheres $(77,97 \%)$ e trabalhadores noturnos $(67,80 \%)$. A distribuição dos dados revela que, proporcionalmente, há mais mulheres trabalhando no turno noturno (Figura $1 \mathrm{~A}, \mathrm{X}^{2}(1,58)=4,588$, $\mathrm{p}=0,032)$. A profissão de Técnico $(66,1 \%)$ é a mais prevalente no estudo. Entretanto, não há preferência para nenhum dos cargos (Figura 1B, $\left.\mathrm{X}^{2}(2,58)=0,881, \mathrm{p}=0,644\right)$. $\mathrm{O}$ quadro profissional revela uma maioria de trabalhadores entre 0-9 anos de serviço $(54,2 \%)$, sendo que não há, também, uma preferência quanto ao turno de trabalho e o tempo na função desempenhada (Figura $1 \mathrm{C}, \mathrm{X}^{2}{ }_{(3,58)}$ $=0,506, \mathrm{p}=0,918$ ).
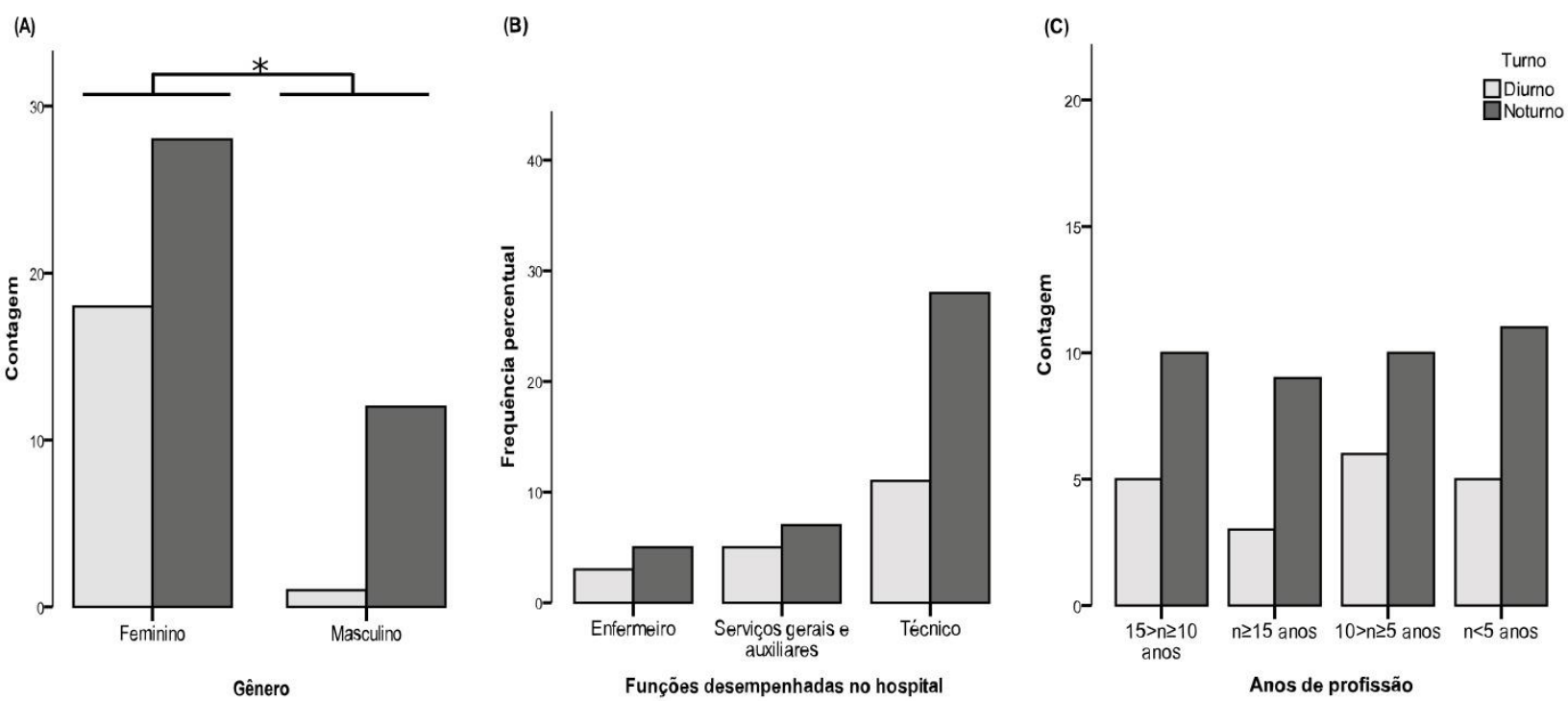

Figura 1. Caracterização dos participantes. (A) Histograma da distribuição de gênero separados por turno de trabalho. Notem que há proporcionalmente mais mulheres no trabalho noturno do que homens. (B) Representação da distribuição dos trabalhadores por profissão. Todos os grupos apresentam distribuição semelhante entre os turnos de trabalho diurno e noturno. (C) Histograma representando o quantitativo de trabalhadores por turno de acordo com o tempo de serviço na função desempenhada. A distribuição entre os turnos é semelhante, independentemente do tempo de serviço $(* p<0,05)$.

Os cronotipos apresentam uma curva de distribuição normal entre trabalhadores, como podemos observar na Figura 2A, que mostra a distribuição da pontuação do questionário $\mathrm{HO}^{23}$. Há uma prevalência de indivíduos intermediários $(\mathrm{n}=59 ; \bar{x}=50,779 \pm 11,564)$, como esperado. Além disso, não há preferência do cronotipo quanto a escolha pelo turno de trabalho (Figura $2 \mathrm{~B}, \mathrm{X}^{2}{ }_{(2,58)}$ $=0,294, \mathrm{p}=0,863$ ), o que significa que o turno é atribuído ao trabalhador independentemente da sua preferência diurna. Quando separamos os trabalhadores pelo cronotipo, notamos que a variância na distribuição dos turnos é igual entre os cronotipos (Figura $2 \mathrm{~B}, \mathrm{~F}_{(2,58)}=0,140, \mathrm{p}=0,869$ ).

Ao verificarmos as médias da qualidade do sono (ANOVA, $\mathrm{F}=0,196, \mathrm{df}=2, \mathrm{p}=0,823$ ) nos distintos cronotipos, agrupados em diurnos e noturnos, notamos que esse padrão é bastante semelhante entre os trabalhadores em turno, essa similitude é verificada na sonolência diurna (ANOVA, $\quad \mathrm{F}=0,379, \quad \mathrm{df}=2, \mathrm{p}=0,686) \quad \mathrm{dos}$ cronotipos agrupados pelo turno. Isso demonstra que os padrões de sono não diferem quanto aos distintos padrões fenotípicos.

A preferência diurna $(\mathrm{HO}, \mathrm{t}=0,894, \mathrm{df}=$ 57, $\mathrm{p}=0,375$ ), a qualidade do sono (Pittsburgh, $\mathrm{t}=$ $1,082, \mathrm{df}=57, \mathrm{p}=0,284)$ e a sonolência durante o dia (Epworth, $\mathrm{t}=0,104$, df $=57, \mathrm{p}=0,917$ ) não diferem, em sua média, entre os trabalhadores noturnos e diurnos. Isso indica que quando isolamos essas variáveis e comparamos os resultados dos indivíduos conforme o turno de trabalho, tanto os que trabalham de dia, quanto os que trabalham à noite, apresentam escores semelhantes quanto aos parâmetros de sono, embora o mesmo não ocorra quando cruzamos as variáveis. Cronotipo não está relacionado nem com a qualidade do sono, nem com a sonolência diurna, como mostram os gráficos de correlação 3A e 3B, respectivamente. Ambos indicam correlação insignificante entre variáveis, isto é, a distribuição dos pontos não segue uma tendência positiva nem negativa. 
(A)

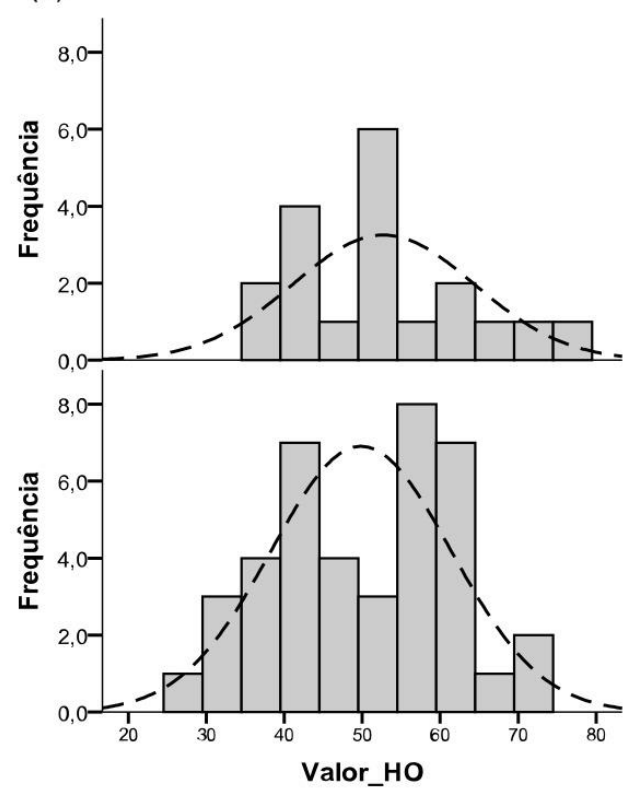

(B)

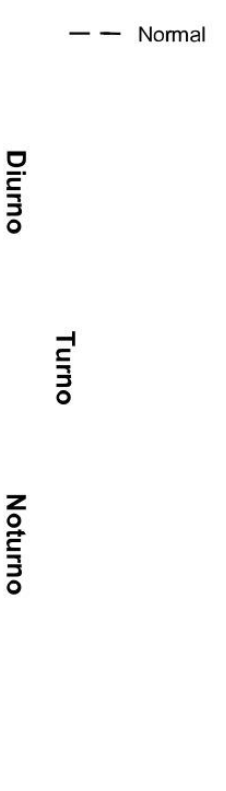

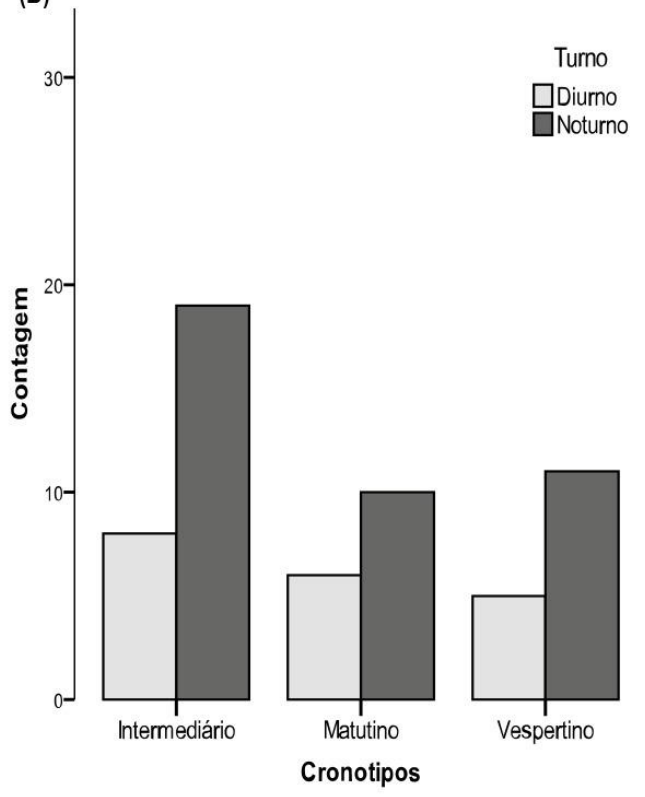

Figura 2. Representação da preferência diurna. (A) O gráfico representa a distribuição da pontuação no questionário HO. Note que há uma curva distribuição normal dos dados para ambos os turnos (linha pontilhada). (B) Histograma da distribuição dos cronotipos dos sujeitos da amostra classificados conforme o turno de trabalho. Note que não há diferença na distribuição do turno dos trabalhadores conforme a preferência diurna.


Figura 3. Correlações entre o cronotipo e os parâmetros de sono. (A) Distribuição dos pontos de correlação entre a preferência diurna (escore HO) versus a qualidade do sono (escore de Pittsburgh) agrupados pelo turno de trabalho. (B) Correlação entre a preferência diurna (escore HO) e a sonolência diurna (escala de Epworth) agrupados pelo turno de trabalho. Os círculos em cinza-claro representam os trabalhadores diurnos e os preenchidos de cinza-escuro, os noturnos. 
Todavia observamos que a distribuição dos escores da qualidade de sono independe da preferência diurna, tanto para os trabalhadores noturnos quanto para os diurnos, revelando que para os trabalhadores de ambos os turnos, a qualidade de sono é semelhante $(n=59, \rho=-0,077$, $\mathrm{p}=0,560 ; \mathrm{R}^{2}$ linear noturno $=0.079, \mathrm{R}_{\text {linear diurno }}^{2}=0,052$, Figura 3A).

Além disso, os cronotipos e a sonolência diurna excessiva não são correlatos mesmo quando agrupadas pelo turno de trabalho $(n=59, \rho=-0,066$, $\mathrm{p}=0,621 ; \mathrm{R}^{2}$ linear noturno $=0,042, \mathrm{R}^{2}$ linear diurno $=0,061$, Figura $3 \mathrm{~B}$ ), reiterando que independe do cronotipo do indivíduo, seu turno de trabalho não interfere na sonolência diurna relatada, logo é semelhante em ambos os grupos.

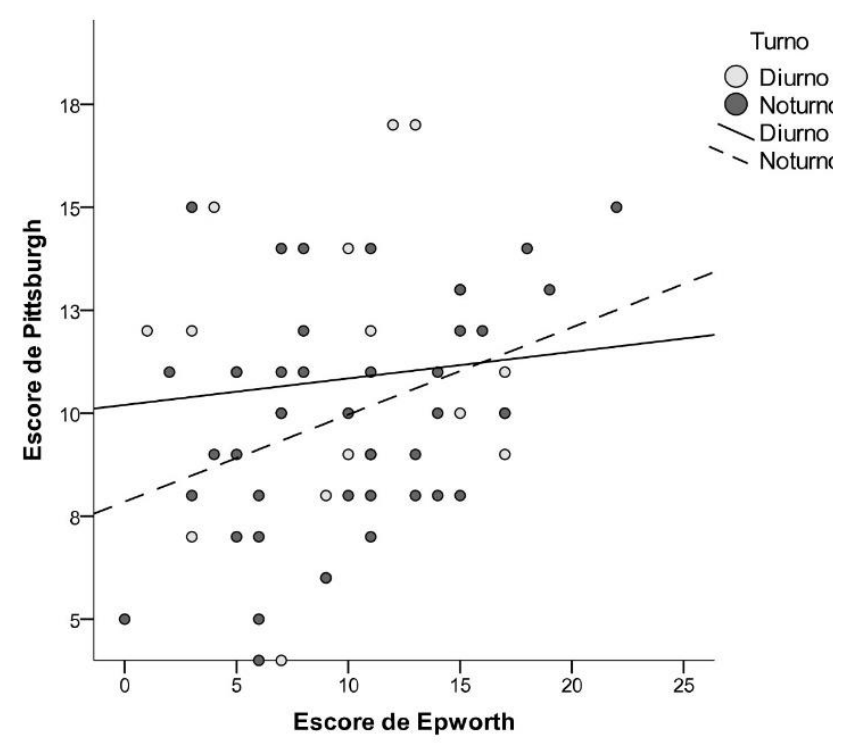

Figura 4. Correlação entre os parâmetros de sono. Há uma correlação positiva entre a qualidade de sono e a sonolência diurna nos trabalhadores noturnos e não está presente entre os trabalhadores diurnos.

Não obstante, o padrão de dispersão dos pontos na figura 4 , por outro lado, revela que nos trabalhadores noturnos há uma correlação positiva entre a sonolência diurna e a qualidade do sono, mostrando que os trabalhadores noturnos apresentam maior sonolência diurna quanto pior sua qualidade de sono, o que não é observado entre os trabalhadores diurnos $(n=59, \rho=0,275, p=0,035$; $\mathrm{R}_{\text {linear noturno }}=0,145, \mathrm{R}_{\text {linear diurno }}=0,009$, Figura 4 ).

\section{DISCUSSÃO}

Verificamos que a maioria dos trabalhadores do hospital universitário do Vale do São Francisco é composta por mulheres, bem como a maioria dos voluntários que respondeu os questionários deste estudo. Uma possibilidade é que a maior parte dos funcionários possui cargo de técnicos e auxiliares no cuidado ao paciente. Historicamente, as mulheres estão mais envolvidas nesse ramo dos profissionais de saúde. A maior parte dos questionários também foi respondida por trabalhadores noturnos, talvez por terem maior interesse na pesquisa e por realizarem o turno proposto neste estudo (12x36h), o que foi utilizado como fator de inclusão.

Ao analisarmos os cronotipos, vimos uma curva de distribuição normal da preferência diurna, o que já era esperado. Entretanto, surpreendentemente, o cronotipo não é levado em consideração para a escolha do turno de trabalho. Esse dado é extremamente relevante, pois reflete uma realidade que deve ser repensada pelos gestores e mesmo pelos trabalhadores. Se o turno não é condizente com a preferência diurna do indivíduo, significa que ele não está trabalhando no horário de sua melhor performance. Isso implica no desempenho deste funcionário, o que é fundamental no caso dos cuidados à saúde da população. É só imaginar um técnico de enfermagem matutino, por exemplo, trabalhando no turno noturno: como estará a sua atenção durante o trabalho? Qual a probabilidade de cometer erros que podem ser cruciais à vida do paciente em questão? Qual a qualidade do sono subsequente ao turno de trabalho? Quando comparadas as taxas de erro de medicação entre os turnos diurno e noturno, a taxa de erro nos turnos noturnos é significativamente maior do que durante o dia ${ }^{26}$. Além disso, há um efeito indireto entre a baixa qualidade e curta duração do sono com o aumento de possíveis erros médicos ${ }^{27}$.

Não é fácil inverter o ritmo de atividade e repouso, pois não implica na mudança do ritmo de uma única variável. Pelo contrário, a maioria (para não dizer quase todas) das variáveis fisiológicas são rítmicas. Apresentam um ritmo circadiano com período de cerca de $24 \mathrm{~h}$. As variáveis fisiológicas possuem relação de fase e interagem entre elas ${ }^{28}$.

Os trabalhadores noturnos precisam inverter a fase ativa, com isso uma série de variáveis também se invertem, como o sono, a atenção, a atividade cerebral, a curva de temperatura corporal, os horários alimentares, entre tantos outros. Porém, não são todos os ritmos que se invertem, como por exemplo a secreção de diversos hormônios. Isso gera uma ruptura das relações de fase entre as variáveis fisiológicas em diversos níveis, restando aos trabalhadores noturnos, se adaptarem a essas mudanças. Além 
disso, no regime de $12 \mathrm{~h}$ de trabalho com 36 horas de folga, os trabalhadores noturnos acabam tendo noites de folga alternadas com noites de trabalho. Com isso, o resultado é uma noite acordados, seguida por uma noite de sono, e assim consecutivamente.

Os efeitos do trabalho noturno não são amplamente difundidos pela sociedade médica. A redução na qualidade e quantidade do sono estão associadas às queixas generalizadas de fadiga, ansiedade, depressão e aumento do neuroticismo ${ }^{29}$. Bem como, evidências crescentes de efeitos cardiovasculares adversos e possível aumento de distúrbios metabólicos relacionados ${ }^{30-32}$.

A qualidade do sono é fundamental para o bom funcionamento do organismo. Quando mantemos um sono saudável, o nosso corpo mantém as taxas de histamina, cortisol, acetilcolina, serotonina e norepinefrina estáveis ${ }^{33-}$ 35. Além disso, o sono atua como um mecanismo neuroprotetor: durante o sono, o cérebro se redefine, removendo subprodutos de resíduos tóxicos que podem ter se acumulado ao longo do $\mathrm{dia}^{36}$. Tais resíduos tóxicos se associam com muitos distúrbios neurodegenerativos, como o acúmulo de proteína beta-amiloide relacionada à doença de Alzheimer, ou acúmulo de proteína tau que pode levar ao aparecimento de graus de demência; destaca-se, portanto, o papel do sono na prevenção de patologias neurodegenerativas ${ }^{37}$. Além disso, desordens comuns do sono, como a insônia, hipersonia ou o transtorno comportamental no sono REM (sigla para Rapid Eye Movement) podem estar presentes no curso da doença de Parkinson ${ }^{38,39}$.

Além do aspecto clínico, uma boa noite de sono pode contribuir para uma melhora da produtividade, relacionando fatores como a memória e raciocínio ${ }^{40-42}$. O sono NREM (NonRapid Eye Movement) atua na consolidação da memória episódica dependente do hipocampo, bem como na memória procedural, isso por aumentar a conectividade sináptica entre o hipocampo e o córtex ${ }^{43,44}$. A ativação de neurônios do córtex retrosplenial (RSC), durante o sono REM, e seu acoplamento com a oscilação teta hipocampal pode estar envolvida na consolidação da memória contextual ${ }^{45}$. Além disso, durante o sono REM, a inibição por optogenética de neurônios GABAérgicos no septo medial, que se projetam para o hipocampo e RSC, implica na redução da potência do ritmo teta, e consequente redução da memória contextual ${ }^{45,46}$.

Análises comparativas demostram que a perda de produtividade devido a um sono insuficiente, dentro do aspecto macroeconômico, pode ser responsável por até US\$ 680 bilhões perdidos ao $\mathrm{ano}^{47}$. Somente nos Estados Unidos, a falta de um sono saudável acarreta um impacto econômico anual entre US\$ 30 a US\$ 40 bilhões devido a custos diretos e indiretos de serviços de saúde $^{48}$. Por exemplo, um indivíduo com apneia obstrutiva do sono não tratada custa $\$ 4261$ a mais do que um indivíduo sem apneia, em termos de acidente, doenças, baixa produtividade e absentismo ${ }^{47}$.

O estudo realizado apresentou limitações quanto à sua população e amostra. No que diz respeito à população definida, alguns indivíduos diurnos e noturnos trabalhavam em outros empregos durante o seu tempo de repouso, isto é, durante as 36 horas solicitadas na jornada $12 \times 36$; embora, no questionário não havia uma pergunta específica que separasse os que trabalham, e os que repousam nessa fase. A amostra pode ser considerada um fator limitante, tendo em vista que, aproximadamente, apenas $10 \%$ do quadro de funcionários do Hospital Universitário do Vale do São Francisco respondeu ao questionário. Desse modo, os resultados encontrados refletem apenas a população em questão.

Estão sendo discutidas algumas medidas para a melhora da qualidade de vida dos trabalhadores em turno pelos programas de saúde do trabalho. Tais como a manipulação da exposição à luz, através do tratamento alternado com luz intensa e bloqueio da luz azul com óculos laranja, a fim de realinhar os ritmos circadianos ${ }^{49}$; delinear individualmente estratégias que reduzem o risco para cada ocupação $0^{50}$; incluir intervalos durante o turno noturno e estabelecer marcadores de fadiga, facilmente identificáveis ${ }^{51}$.

Implementando algumas estratégias simples, poderemos trabalhar na melhor performance para os profissionais e para os atendidos. Estratégias como o treinamento da equipe, no sentido de conscientizá-los sobre seus próprios ritmos biológicos, alertando-os de como podem se organizar e tomar medidas comportamentais que os alertem sobre os sinais de fadiga, tanto próprios quanto de seus colegas, indicando que algo não está indo bem. Além de prezar pela qualidade de vida dos trabalhadores, auxiliará no gerenciamento de ações preventivas e eficazes. Todas essas medidas visam manter os trabalhadores em turnos saudáveis e a salvo de possíveis erros. 


\section{CONCLUSÃO}

A necessidade do funcionamento ininterrupto dos serviços de saúde demanda a disposição de que alguns indivíduos estejam aptos a trabalhar nos turnos noturnos, invertendo o ritmo circadiano de atividade e repouso. Isso gera uma ruptura das relações de fase entre as variáveis fisiológicas em diversos níveis, restando aos trabalhadores noturnos, se adaptarem a essas mudanças. Desconsiderar as variáveis biológicas no aspecto social pode ocasionar riscos, sobretudo quando os que a realizam estão intrinsecamente relacionados com o cuidado à vida alheia. Isso destaca que a preferência diurna é uma característica essencial na escolha do turno, embora nesse estudo observamos que a preferência não foi determinante para a escolha do turno de trabalho. Em todos cronotipos os trabalhadores apresentaram a mesma qualidade de sono e a mesma sonolência diurna. Porém, em profisionais noturnos quanto pior for a sua qualidade do sono, maior a sonolência relatada, o que leva a um maior o risco de ocorrer erros, algo que não é observado em profissionais diurnos.

\section{AGRADECIMENTOS}

Agradecemos a todos os voluntários que responderam a esta pesquisa, aos estudantes envolvidos na coleta de dados, ao Hospital Universitário da UNIVASF que permitiu o acesso aos funcionários, à Fundação de Amparo à Pesquisa do Estado de Alagoas (FAPEAL) e ao programa de Bolsas de Incentivo Acadêmico da Universidade Federal do Vale do São Francisco pelo suporte financeiro. Agradecimento especial ao professor Dr. John Fontenele Araújo pelas suas preciosas sugestões.

\section{REFERÊNCIAS}

1. Koenigsberg HW, Teicher MH, Mitropoulou V, Navalta C, New AS, Trestman R, et al. 24-h Monitoring of plasma norepinephrine, MHPG, cortisol, growth hormone and prolactin in depression. $\mathbf{J}$ Psychiatr Res 2004;38:503-11.

https://doi.org/10.1016/j.jpsychires.200 4.03.006
2. Báez-Ruiz A, Guerrero-Vargas NN, Cázarez-Márquez F, Sabath E, Basualdo MDC, Salgado-Delgado R, et al. Food in synchrony with melatonin and corticosterone relieves constant light disturbed metabolism. J Endocrinol 2017;235:167-78. https://doi.org/10.1530/JOE-17-0370.

3. Heilbronn LK, Regmi P. Will Delaying Breakfast Mitigate the Metabolic Health Benefits of Time-Restricted Eating? Obesity 2020;28. https://doi.org/10.1002/oby.22776.

4. Pereira DS, Tufik S, Pedrazzoli M. Moléculas que marcam o tempo: implicações para os fenótipos circadianos. Rev Bras Psiquiatr 2009;31:63-71. https://doi.org/10.1590/S151644462009000100015.

5. Ferguson B, Shoff H, Shreffler J, McGowan J, Huecker M. Does My Emergency Department Doctor Sleep? The Trouble With Recovery From Night Shift. J Emerg Med 2019;57:1627.

https://doi.org/10.1016/j.jemermed.201 9.04.023.

6. Smarr BL, Schwartz MD, Wotus C, de la Iglesia HO. Re-examining "temporal niche". Integr Comp Biol 2013;53:16574. https://doi.org/10.1093/icb/ict055.

7. Roenneberg T, Merrow M. Entrainment of the human circadian clock. Cold Spring Harb Symp Quant Biol 2007;72:293-9. https://doi.org/10.1101/sqb.2007.72.04 3.

8. Vetter C. Circadian disruption: What do we actually mean? Eur J Neurosci 2020;51:531-50. https://doi.org/10.1111/ejn.14255.

9. Boivin DB, Boudreau P, James FO, Kin NM. Photic resetting in night-shift work: impact on nurses' sleep. 
Chronobiolology Int 2012;29:619-28. https://doi.org/10.3109/07420528.2012 .675257 .

10. HALBERG F. Some physiological and clinical aspects of 24-hour periodicity. $\mathrm{J}$ Lancet 1953;73:20-32.

11. Abbott SM, Malkani RG, Zee PC. Circadian disruption and human health: A bidirectional relationship. Eur J Neurosci 2020;51:567-83. https://doi.org/10.1111/ejn.14298.

12. Honma K, Hashimoto S, Nakao M, Honma S. Period and Phase Adjustments of Human Circadian Rhythms in the Real World. J Biol Rhythms 2003;18:261-70. https://doi.org/10.1177/074873040301 8003008 .

13. Azzi A, Evans JA, Leise T, Myung J, Takumi T, Davidson AJ, et al. Network Dynamics Mediate Circadian Clock Plasticity. Neuron 2017;93:441-50. https://doi.org/10.1016/j.neuron.2016.1 2.022 .

14. Liu AC, Lewis WG, Kay SA. Mammalian circadian signaling networks and therapeutic targets. Nat Chem Biol 2007;3:630-9. https://doi.org/10.1038/nchembio.2007. 37.

15. Koike BDV. Rastreamento de polimorfismos no gene AANAT e suas associações com a preferência diurna. Universidade Federal de São Paulo (UNIFESP), 2009.

16. Richter HG, Torres-Farfń C, RojasGarcía PP, Campino C, Torrealba F, Serón-Ferré $M$. The circadian timing system: Making sense of day/night gene expression. Biol Res 2004;37:11-28. https://doi.org/10.4067/S071697602004000100003.

17. Beersma DGM, Gordijn MCM. Circadian control of the sleep-wake cycle. Physiol Behav 2007;90:190-5. https://doi.org/https://doi.org/10.1016/j. physbeh.2006.09.010.

18. Folkard S, Lombardi DA. Modeling the impact of the components of long work hours on injuries and "accidents." Am J Ind Med 2006;49:953-63.

https://doi.org/10.1002/ajim.20307.

19. Daan S, Beersma DG, Borbely AA. Timing of human sleep: recovery process gated by a circadian pacemaker. Am J Physiol Integr Comp Physiol 1984;246:R161-83.

https://doi.org/10.1152/ajpregu.1984.2 46.2.R161.

20. Roenneberg T, Merrow M. The Circadian Clock and Human Health. Curr Biol 2016;26:R432-43. https://doi.org/10.1016/j.cub.2016.04.0 11.

21. Benedito-Silva AA, Menna-Barreto L, Marques N, Tenreiro S. A selfassessment questionnaire for the determination of morningnesseveningness types in Brazil. Prog Clin Biol Res 1990;341 B:89-98.

22. Menna-Barreto L. O tempo na biologia. Cronobiologia Princípios e Apl 2003:26-9.

23. Horne JA, Ostberg O. A self-assessment questionnaire to determine morningness-eveningness in human circadian rhythms. Int $\mathrm{J}$ Chronobiol 1976;4:97-110.

24. Johns MW. A new method for measuring daytime sleepiness: The Epworth sleepiness scale. Sleep 1991;14:540-5. https://doi.org/10.1093/sleep/14.6.540.

25. Buysse DJ, Reynolds CF, Monk TH, Berman SR, Kupfer DJ. The Pittsburgh sleep quality index: A new instrument for psychiatric practice and research. Psychiatry Res 1989;28:193-213. 
https://doi.org/10.1016/01651781(89)90047-4.

26. Miller AD, Piro CC, Rudisill CN, Bookstaver PB, Bair JD, Bennett CL. Nighttime and weekend medication error rates in an inpatient pediatric population. Ann Pharmacother 2010. https://doi.org/10.1345/aph.1P252.

27. Kalmbach DA, Arnedt JT, Song PX, Guille C, Sen S. Sleep disturbance and short sleep as risk factors for depression and perceived medical errors in firstyear residents. Sleep 2017;40:1-8. https://doi.org/10.1093/sleep/zsw073.

28. Golombek D a, Aguilar-Roblero R. Mecanismos de Temporização em vertebrados. In: Marques N, MennaBarreto L, editors. Cronobiologia Princípios e Apl. $3^{\text {a }}$, São Paulo: Editora da Universidade São Paulo -; 2003, p. 137-62.

29. Harrington JM. Health effects of shift work and extended hours of work. Occup Environ Med 2001;58:68-72. https://doi.org/10.1136/oem.58.1.68.

30. Li JZ, Bunney BG, Meng F, Hagenauer MH, Walsh DM, Vawter MP. Circadian patterns of gene expression in the human brain and disruption in major depressive disorder. Pnas 2013;110:9950-5. https://doi.org/10.1073/pnas.13058141 $10 /-$

/DCSupplemental.www.pnas.org/cgi/d oi/10.1073/pnas.1305814110.

31. de Oliveira IGB, Junior MDF, Lopes PR, Campos DBT, Ferreira-Neto ML, Santos EHR, et al. Forced internal desynchrony induces cardiometabolic alterations in adult rats. $\mathrm{J}$ Endocrinol 2019;242:25-36.

https://doi.org/10.1530/JOE-19-0026.

32. Bass J, Takahashi JS. Circadian integration of metabolism and energetics. Science (80- )
2010;330:1349-54.

https://doi.org/10.1126/science.119502 7.

33. Swick TJ. The Neurology of Sleep: 2012. Sleep Med Clin 2012;7:399-415. https://doi.org/10.1016/j.jsmc.2012.06. 003.

34. Pantazopoulos H, Gamble K, Stork O, Amir S. Circadian Rhythms in Regulation of Brain Processes and Role in Psychiatric Disorders. vol. 2018. 2018.

35. Wesermann W, Roysch, Schulz, Zofel. Circadian rhythm of serotonin binding in rat brain- 1986;3:135-9.

36. Bunney PE, Zink AN, Holm AA, Billington $\mathrm{CJ}$, Kotz CM. Orexin activation counteracts decreases in nonexercise activity thermogenesis (NEAT) caused by high-fat diet. Physiol Behav 2017;176:139-48. https://doi.org/10.1016/j.physbeh.2017. 03.040 .

37. Abbott SM. Chronic sleep disturbance and neural injury 2016:55-61.

38. Lees AJ, Blackburn NA, Campbell VL. The nighttime problems of Parkinson's disease. Clin Neuropharmacol 1988. https://doi.org/10.1097/00002826198812000-00004.

39. Tandberg E, Larsen JP, Karlsen K. A community-based study of sleep disorders in patients with Parkinson's disease. Mov Disord 1998. https://doi.org/10.1002/mds.870130606

40. Poe G, Walsh C, Bjorness T. Both duration and timing of sleep are important to memory consolidation. Sleep 2010;33:1315-22.

41. Dijk D-J, von Schantz M. Timing and consolidation of human sleep, wakefulness, and performance by a 
symphony of oscillators. J Biol Rhythms 2005;20:279-90. https://doi.org/10.1177/074873040527 8292.

42. Siegel JM. The REM sleep-memory consolidation hypothesis. Science 2001;294:1058-63. https://doi.org/10.1126/science.106304 9.

43. Acosta MT. Sleep, memory and learning. Medicina (B Aires) 2019;79:29-32.

44. Ackermann S, Rasch B. Differential effects of non-REM and REM sleep on memory consolidation? Curr Neurol Neurosci Rep 2014. https://doi.org/10.1007/s11910-0130430-8.

45. Koike BDV, Farias KS, Billwiller F, Almeida-Filho D, Libourel P-A, TiranCappello A, et al. Electrophysiological Evidence That the Retrosplenial Cortex Displays a Strong and Specific Activation Phased with Hippocampal Theta during Paradoxical (REM) Sleep. J Neurosci 2017;37:8003-13. https://doi.org/10.1523/JNEUROSCI.0 026-17.2017.

46. Boyce R, Glasgow SD, Williams S. Casual evidence for the role of REM sleep theta rhythm in contexual memory consolidaiton. Science (80- ) 2016.

47. Chilcott LA, Shapiro CM. The socioeconomic impact of insomnia: An overview. Pharmacoeconomics, 1996. https://doi.org/10.2165/00019053199600101-00003.
48. Hafner M, Stepanek M, Taylor J, Troxel W, Stolk C. Why sleep matters -- the economic costs of insufficient sleep: A cross-country comparative analysis. 2017. https://doi.org/10.7249/rr1791.

49. Lowden A, Öztürk G, Reynolds A, Bjorvatn B. Working Time Society consensus statements: Evidence based interventions using light to improve circadian adaptation to working hours. Ind Health 2019;57:213-27. https://doi.org/10.2486/indhealth.SW9.

50. Gärtner J, Rosa RR, Roach G, Kubo T, Takahashi M. Working Time Society consensus statements: Regulatory approaches to reduce risks associated with shift work - a global comparison. Ind Health 2019;57:245-63. https://doi.org/10.2486/indhealth.SW7.

51. Wong IS, Popkin S, Folkard S. Working Time Society consensus statements: A multi-level approach to managing occupational sleep-related fatigue. Ind Health 2019;57:228-44. https://doi.org/10.2486/indhealth.SW6. 